Esta revista forma parte del acervo de la Biblioteca Jurídica Virtual del Instituto de Investigaciones Jurídicas de la UNAM http://www.juridicas.unam.mx 


\title{
Teorías relativas al cumplimiento del derecho internacional público
}

\author{
Theories about Compliance with Public \\ International Law
}

\section{Théories sur la conformité au droit international public}

\begin{abstract}
María Helena Carbonell Yánez*
SUMARIO: I. Introducción. II. Teorías tradicionales del derecho internacional. III. Nuevos enfoques teóricos del derecho internacional público. IV. Conclusión. V. Bibliografía.
\end{abstract}

* Instituto de Altos Estudios Nacionales, Ecuador; ORCID ID: https: / / orcid.org/0000-00 03-0114-7592,maria.helena.carbonell@gmail.com.

Artículo recibido el 12 de septiembre de 2019 Aprobado para publicación el 9 de octubre de 2020 
Resumen: Como bien lo señalaba Henkin, “casi todas las naciones observan casi todos los principios de derecho internacional y casi todas sus obligaciones casi todo el tiempo". Esto significa que los casos de incumplimiento son una excepción, que podrá ser sometida al conocimiento de un tribunal internacional, para que sea éste quien dirima la controversia, creando nuevas obligaciones para el o los Estados intervinientes. En este panorama, la pregunta que guía esta investigación es cuáles han sido las respuestas que las diferentes teorías filosóficas del derecho internacional han dado a la pregunta de por qué los Estados cumplen con estas obligaciones. Desde un abordaje histórico, este trabajo pretende dar al lector/a una sistematización de los principales aportes desde el campo jurídico, así como de las relaciones internacionales.

Palabras clave: derecho internacional público, cumplimiento, obligaciones internacionales, relaciones internacionales.

ABSTRACT: As Henkin pointed out, "almost all nations observe almost all principles of international law and almost all of their obligations almost all of the time". This implies that non-compliance is an exception, that can be brought before an international tribunal/court so that any controversy can be solved. This means that new obligations for the intervening State or States are created though the judgment delivered by the court. This paper addresses what has been the answer that the main philosophical international law theories have given to the question of why States comply with international law. Using a historical approach, this paper offers the reader a comprehensive systematization of the main contributions made not only by the legal theories, but also by those embedded in the international relations field.

Key words: public international law, compliance, international obligations, international relations.

RÉSUMÉ: Commme l'a bien noté Henkin, "presque toutes les nations observen presque tous les principes du droit international et presque toutes leurs obligations presque tout le temps". Cela signifie que les cas de violation sont une exception, qui pourra être soummise à l'examen d'un tribunal international, afin que celui-ci résolve le différend à travers la création de nouvelles obligations pour le ou les États intervenants. Dans cette optique, cette reherche porte sur les réponses que les diferentes théories philosophiques du droit international offrent à la question de savoir pourquoi les États remplissent ces obligations. A partir d'une approche historique, ce travail prétend offrir aux lecteurs une systématisation des principaux apports du champ juridique ainsi que des relations internationales.

Mots-clés: droit international public, conformité, obligations internationales, relations internationales. 


\section{INTRODUCCIÓN}

Henkin afirma que "casi todas las naciones observan casi todos los principios de derecho internacional y casi todas sus obligaciones casi todo el tiempo". ${ }^{1}$ De acuerdo con el autor, el incumplimiento de las obligaciones internacionales del Estado es la excepción frente a un cumplimiento, que es la regla en las relaciones interestatales. Si el panorama es como lo plantea Henkin, la pregunta que se debe plantear es por qué los Estados — estas entidades casi todopoderosas en el plano internacional — cumplen con sus obligaciones. En caso de incumplimiento, los tribunales internacionales, entendidos como órganos permanentes, habiendo sido creados por un acuerdo internacional, recurren al derecho internacional para resolver los conflictos que les sean presentados. Esto lo hacen a través de un conjunto de reglas previamente establecidas (que las partes no pueden modificar) y emiten una decisión obligatoria para las partes intervinientes. ${ }^{2}$ Adicionalmente a los tratados, la costumbre, los principios generales y los actos unilaterales, las sentencias emitidas por dichos tribunales internacionales son de obligatorio cumplimiento para las partes, ya que contienen obligaciones dirigidas al Estado o Estados responsables.

A pesar de que los Estados son los principales destinatarios y creadores de las normas de derecho internacional, el panorama actual demuestra que en las relaciones internacionales intervienen otros actores, incluyendo en el contencioso internacional. Los tribunales, al resolver controversias no in-

1 Henkin, Louis, How Nations Behave: Law and Foreign Policy, 2a. ed., Nueva York, Columbia University Press, 1979, p. 26.

2 Alter, Karen, "Agents or Trustees? International Courts in their Political Context", European Journal of International Relations, Nueva York, vol. 14, núm. 1, 2008, p. 34; Romano, Cesare, "The Proliferation of International Judicial Bodies: The Pieces of the Puzzle", New York University Journal of International Law and Politics, Nueva York, vol. 31, núm. 4, 1999, p. 715; Sorensen, Max, Manual de derecho internacional público, trad. de Dotación Carnegie para la Paz Internacional, México, Fondo de Cultura Económica, 2011, p. 179; Tomuschat, Christian, "International Courts and Tribunals", en Rüdiger, Wolfrum (ed.), Max Planck Encyclopedia of Public International Law, Reino Unido, Oxford University Press, 2008, disponible en: https: / / opil.ouplaw.com/view/10.1093/law:epil/9780199231690/law-9780199231690-e35?rskey=Wj $Z_{w} R 8$ \&result $=6 \& p r d=M P I L$; Voeten, Erik, "International Judicial Independence”, en Dunoff, Jeffrey y Pollack, Mark A. (eds.), Interdisciplinary Perspectives on International Law and International Relations: The State of the Art, Reino Unido, Cambridge University Press, 2012, p. 422. 
terestatales, también determinan la responsabilidad internacional del Estado y su obligación de reparar. Siguiendo el planteamiento de Henkin, varios estudios han demostrado que los Estados cumplen (pero no en su totalidad) las obligaciones provenientes de las sentencias de los tribunales de derechos humanos. $^{3}$

Aunque las principales teorías relativas al cumplimiento del derecho internacional hacen referencia a los tratados (como fuente de obligaciones), es posible extrapolar sus conclusiones al cumplimiento de las decisiones provenientes de órganos jurisdiccionales. Este trabajo plantea verificar cómo las diferentes teorías intentan explicar por qué los Estados cumplen con dichas obligaciones internacionales, enfocándose en el tratamiento de relaciones claramente asimétricas entre dos sujetos del derecho internacional: la persona y el Estado.

En las páginas siguientes, el estudio sigue una línea cronológica, partiendo de las teorías que hemos denominado "tradicionales" o "clásicas", que abarcan desde los negacionistas, al positivismo y a las teorías del proceso transnacional. En un segundo capítulo se estudian aquellas "nuevas teorías", que comprenden los Critical Legal Studies y los aportes del tercer mundo al derecho internacional. Esta revisión teórico-cronológica sobre los motivos que explican la adecuación de la conducta del Estado a sus obligaciones internacionales es aplicada al caso de las sentencias provenientes de tribunales que declaran la responsabilidad del Estado por violaciones a los derechos humanos.

3 Baluarte, David C., "Strategizing for Compliance: The Evolution of a Compliance Phase of Inter-American Court Litigation and the Strategic Imperative for Victims' Representatives", American University International Law Review, Washington D. C., vol. 27, núm. 2, 2012, pp. 263 319; Hawkins, Darren y Jacoby, Wade, "Partial Compliance: A Comparison of the European and Inter-American Courts of Human Rights", Journal of International Law and International Relations, Toronto, vol. 6, núm. 1, 2010, pp. 35-85; Huneeus, Alexandra, "Compliance with International Court Judgments and Decisions”, en Romano, Cesare et al. (eds.), The Oxford Handbook of International Adjudication, Reino Unido, Oxford University Press, 2015, pp. 348463; Poertner, Mathias, "Institutional Capacity for Compliance: Domestic Compliance with the Inter-American Court of Human Rights”, APSA 2013 Annual Meeting Paper, Chicago, 2013, pp. 1-33, disponible en: https://ssrn.com/abstract=2299763; Posner, Eric A. y Yoo, John C., "Judicial Independence in International Tribunals", California Law Review, Berkeley, vol. 93, núm. 1, 2005, pp. 3-72; Romero, Alexia y Staton, Jeffrey K., "Clarity and Compliance in the Inter-American Human Rights System” (manuscrito), 2011, pp. 1-30, disponible en: http: / biblioteca.cejamericas.org/bitstream / handle/2015/448/Clarity-and-Compliance-in-the-Inter-Ameri can-Human-Rights-System.pdf?sequence $=1$ \&isAllowed $=y$. 


\section{TEORÍAS TRADICIONALES DEL DERECHO INTERNACIONAL}

La revisión de las teorías clásicas del derecho internacional (en adelante, el "DI") se hace en función de un estudio histórico de las mismas, identificando rasgos comunes con relación a las razones que se plantean para explicar el cumplimiento de las obligaciones provenientes de los fallos de tribunales en materia de derechos humanos. La primera mirada hacia el DI consistía en negar su carácter de derecho, ya que estaba basada en su equiparación al derecho interno: al no cumplir con los elementos que este último tiene (en función de los actores, sus expectativas y las estructuras jurídicas), se argumentaba que no era posible considerarlo como derecho. ${ }^{4}$ Incluso, Hart y Austin, quienes afirman que el DI no cuenta con todos los elementos del derecho y no es, por lo tanto, un ordenamiento jurídico bien desarrollado, admiten que influencia en el comportamiento del Estado. ${ }^{5}$

En la actualidad, esta teoría ya no cuenta con un gran número de adeptos, al aceptar al DI como una rama del derecho que regula las relaciones internacionales entre sus sujetos. ${ }^{6}$

\section{Teorías jurídicas}

Una vez que se dejan de lado las teorías "negacionistas" del DI, las teorías jurídicas tradicionales están basadas en la concepción de éste como un sistema de normas objetivas y neutrales que emanan de la voluntad del Estado, siendo estos últimos sus actores fundamentales. Grocio, en su búsqueda de la "cientifización" y secularización del derecho, afirma que el DI no tiene un fundamento teológico, convirtiendo al ius gentium en universal (y no propio

4 Herdegen, Matthias, Derecho internacional público, trad. de Marcela Anzola, México, Fundación Konrad Adenauer-UNAM, Instituto de Investigaciones Jurídicas, 2005, pp. 8-11.

5 Austin, John, The Providence of Jurisprudence Determined, Reino Unido, Cambridge University Press, 2010, pp. 132 y 145; Hart, Herbert, El concepto del derecho, trad. de Genaro R. Carrió, Buenos Aires, Abeledo-Perrot, 1963, pp. 263-265.

6 McDougal, Myres S. et al., "Theories about International Law: Prologue to a Configurative Jurisprudence”, Faculty Scholarship Series, Estados Unidos, núm. 2577, 1968, pp. 208-214, disponible en: http://digitalcommons.law.yale.edu/fss_papers/2577. 
de las naciones cristianas). ${ }^{7}$ Las normas internacionales, en este paradigma, deben cumplir con el test de validez otorgado por lo que Bianchi denomina un "pedigrí formal". ${ }^{8}$ Gana importancia, entonces, el concepto de autoridad, sin importar su origen (deidad, presupuestos físicos o el propio ser humano), ${ }^{9}$ ya que es ésta la que le da validez a la norma y, por ende, al sistema.

En vista de la centralidad de la norma, las consideraciones extralegales quedan fuera del análisis jurídico y sus defensores/as rechazan acercamientos teóricos que se consideren "muy alejados de la práctica".${ }^{10}$ En consecuencia, el trazar fronteras es esencial para el análisis del fenómeno legal, por lo que se construye un sistema de análisis dicotomizado y jerarquizado. Esto acarrea tres consecuencias. La primera hace referencia a la creación de fronteras fijas de normatividad que permiten hablar de "ley" (con carácter obligatorio) y "no ley" (no obligatoria). ${ }^{11}$ Este presupuesto es fundamental para el positivismo jurídico en el DI, pero no es exclusivo de esta corriente: incluso aquellas vertientes encasilladas en el marco de la ley natural establecen dicotomías. En este último caso, la dualidad está entre un derecho positivo que, según sus críticos, no alcanza a explicar el DI y un "orden natural general que deriva de las regularidades físicas del mundo físico, de la uniformidad de la psique humana o de una deidad omnipotente". ${ }^{12}$

La segunda consecuencia mencionada por Bianchi es la creación de una frontera esencial entre "lo legal" y "lo no legal”, descartando este último de la actividad judicial. La tercera consecuencia tiene que ver con la metodología para determinar la validez de una norma. En las teorías tradicionales existen criterios de validez dentro del propio DI que están basados en la noción de autonomía del derecho y de rigidez del fenómeno jurídico. Para Bianchi, esta dicotomización se acompaña de un proceso de racionalización y sistematización que busca curar el caos que nace con la existencia de materiales "crudos" (como las sentencias) que no serían propiamente fuentes del DI. Ya que el derecho se presenta como racional, el trabajo de los abogados y

7 Grotius, Hugo, The Rights ofWar and Peace, trad. de Archibald Campbell, Nueva York-Londres, M. Walter Dunne, 2014, cap. III.

8 Bianchi, Andrea, International Law Theories. An Inquiry into Different Ways of Thinking, Reino Unido, Oxford University Press, 2016, p. 24.

9 McDougal, Myres S. et al., op. cit., p. 222.

10 Bianchi, Andrea, op. cit., p. 22.

11 Ibidem, p. 24.

12 McDougal, Myres S. et al., op. cit., p. 218. 
abogadas es sistematizar ese conjunto de materiales "crudos" para construir un sistema coherente (alejándose de la fragmentación) que permita explicar el mundo exterior. ${ }^{13}$

Dentro de las teorías tradicionales se encuentran, asimismo, aquellas que, aunque sin alejarse del formalismo clásico, intentan extender el alcance teórico del DI. Mediante la analogía con el derecho interno (entendido como el ordenamiento jurídico propio de las democracias liberales), a través de la creación de estructuras cuasiconstitucionales, el internacionalismo constitucional mantiene una jerarquización/verticalización de las normas (por ejemplo, el ius cogens es considerado como norma suprema en el ámbito internacional), de los actores internacionales (por ejemplo, la ONU como máxima expresión de una organización supranacional $)^{14}$ y de los valores de la comunidad internacional (habrá valores que esta comunidad considera como esenciales y que merecen una protección internacional). El objetivo de estas creaciones es liberar al DI de lo político (entendiendo que este último "puede domesticarse"), sometiendo al mundo a un imperio de la ley limitado (se mantienen el principio de subsidiariedad, la proporcionalidad y el margen de apreciación que da preferencia a lo local frente a lo internacional), y, de esta manera, proteger a las personas de la inseguridad jurídica.

Mediante su proliferación, las cortes internacionales de derechos humanos parecen convertirse en garantías suprajurisdiccionales (y, en algunos niveles, supra-cuasijurisdiccionales) para hacer cumplir esos valores fundamentales de la comunidad internacional. ${ }^{15}$ Sobre la importancia del trabajo de los órganos internacionales de protección de derechos humanos, la Corte Internacional de Justicia, en el caso Ahmadou Sadio Diallo, parece inclinarse por respetar la interpretación que hayan hecho en el marco de sus competencias. ${ }^{16}$ Esta idea de una soberanía estatal más humana es uno de los prin-

13 Bianchi, Andrea, op. cit., pp. 27, 28 y 30-33; Grotius, Hugo, op. cit.; McDougal, Myres S. et al., op. cit., p. 218.

14 Klabbers, Jan, “Constitutionalism and the Making of International Law Fuller's Procedural Natural Law”, No Foundations: Journal of Extreme Legal Positivism, Helsinki, núm. 5, 2008, pp. 84-112.

15 Aparicio, Marco y Pisarello, Gerardo, "Los derechos humanos y sus garantías", en Bonet Pérez, Jordi y Sánchez, Víctor (coords.), Los derechos humanos en el siglo XXI. Continuidad y cambios, España, Huygens, 2008; Bianchi, Andrea, op. cit., pp. 49-69.

16 Corte Internacional de Justicia, Caso Ahmadou Sadio Diallo (República de Guinea vs. República Democrática de Congo), 30 de noviembre de 2010, párrs. 66-70. 
cipales aportes de esta teoría, pero no llega a matizarse en la realidad frente a las decisiones de estos órganos.

A pesar de la incorporación de nuevos sujetos internacionales, para estas teorías, el Estado sigue siendo un actor fundamental (la persona es un actor importante, pero no son teorías man-oriented) y mantienen diferencias (no siempre claras) entre el derecho interno y el DI. La comunidad internacional aparece como un conjunto de Estados separados que sólo trabajan en conjunto para la protección de valores que se consideran fundamentales y que no necesariamente corresponderán con el respeto y garantía de los derechos humanos. ${ }^{17}$

Desde la escuela de New Haven, la teoría del proceso transnacional (transnational legal process, en inglés) propone una construcción teórica que hace que la línea entre derecho nacional y DI se haga cada vez más borrosa (al igual que en la corriente del derecho administrativo global). Las normas internacionales se cumplen porque existe un proceso de internación que implicaría su domesticación (hacerlas domésticas). Esto podría verificarse, por ejemplo, en los procesos de reconocimiento de derechos en las Constituciones. En este caso, el proceso se logra a través de la interacción de diferentes grupos nacionales que buscan la internación (a través de la adopción o de cierta interpretación de tal o cual norma). Se hablará, entonces, de un solo cuerpo transnacional de normas resultante de un proceso en tres etapas: interacción, interpretación e internación. ${ }^{18}$ Adicionalmente, se concreta una tendencia a la interdisciplinariedad (que se consolidará en las corrientes críticas), a través del uso de herramientas metodológicas extrajurídicas y permitiendo una fertilización cruzada con otras ramas del conocimiento. Sin embargo, se mantienen rasgos de las corrientes más tradicionales, como son el énfasis en el Estado y el normativismo.

Para estas teorías tradicionales, en el DI antiguo o primitivo, la pregunta de por qué se debe obedecer/cumplir con las normas internacionales recibe una respuesta fundada en razones "semiteológicas". Posteriormente, surge una respuesta que pone énfasis en el carácter volitivo del DI: el Estado cumplirá con aquellas normas en cuyo proceso de formación manifestó su voluntad (positivismo). ${ }^{19}$ La voluntad del Estado es esencial y se evidencia

17 McDougal, Myres S. et al., op. cit., pp. 224 y 227-250.

18 Bianchi, Andrea, op. cit., pp. 91-109; Koh, Harold, "Why Do Nations Obey International Law?”, The Yale Law Journal, Estados Unidos, vol. 106, núm. 8, 1997, pp. 2645 y 2646.

19 Grotius, Hugo, op. cit.; Koh, Harold, op. cit., pp. 2603-2606 y 2608. 
la tendencia estatal de mantener cierta soberanía en la implementación del DI, incluso al tratarse de sentencias internacionales de carácter obligatorio. ${ }^{20}$ En este marco entra la teoría del consentimiento, que está fundamentada en la idea de que los Estados están obligados a cumplir con aquello a lo que libremente se comprometieron. Este fundamento acompaña las principales teorías clásicas del DI a lo largo del siglo XVII y hasta principios del siglo $\mathrm{XX}$. De igual manera, es posible encontrarlo en la Convención de Viena sobre el Derecho de los Tratados (artículo 26) al recoger el principio de pacta sunt servanda. La Corte Permanente de Justicia Internacional, en el caso del S. S. "Lotus", recogió este planteamiento, al señalar que las reglas que obligan a los Estados emanan de su propia voluntad. ${ }^{21}$ Pero esta teoría no explica el porqué de ese cumplimiento, sino que presume que toda norma internacional será cumplida.

\section{Teorías de las relaciones internacionales}

La interacción de las relaciones internacionales (en adelante, las "RI") y el derecho no llega a ser un diálogo realmente interdisciplinario constante, sino que hay esfuerzos esporádicos que se enfocan en áreas relativas al diseño legal e institucional, a la complejidad de los regímenes (lo que en la doctrina legal corresponde a la fragmentación del DI) y al cumplimiento y eficacia del DI. ${ }^{22}$ En cuanto a las teorías de las RI sobre el DI, la mayoría de éstas enfocan su estudio en la actuación del Estado sin poner énfasis en el rol de los tribunales internacionales en el cumplimiento. ${ }^{23}$ En este apartado se presenta una revisión de las principales teorías de las RI, explicando su postura frente al cumplimiento del DI.

Por un lado, el realismo propugna que el Estado es el principal actor en el plano internacional y se le concibe como un ente racional. Y, como tal,

20 Palombino, Fulvio Maria, “Compliance with International Judgments: Between Supremacy of International Law and National Fundamental Principles”, Zeitschrift für ausländisches öffentliches Recht undVölkerrecht, Heidelberg, núm. 75, 2015, pp. 506, 507 y 510.

21 Corte Permanente de Justicia Internacional, Caso del S. S. "Lotus", 7 de septiembre de 1927.

22 McDougal, Myres S. et al., op. cit., p. 122.

23 Velasco, Shelom, "The Inter-American Court of Human Rights: Emerging Patterns in Judgment Compliance”, Thesis and Dissertations, Bloomington, núm. 29, 2016, pp. 30-43, disponible en: https: / / www.repository.law.indiana.edu/cgi / viewcontent.cgi?article=1028\&context=etd. 
éste siempre elegirá aquellas conductas que aseguren su supervivencia y su crecimiento, entendidos como sus principales intereses. ${ }^{24}$ En consecuencia, el DI tiene poca o casi nula influencia en la conducta de los Estados, ya que éstos cumplen con sus obligaciones cuando es funcional para alcanzar sus intereses (su supervivencia y su crecimiento, entendidos como una acumulación de poder en el marco de la comunidad internacional). ${ }^{25}$ Guzmán, por su lado, afirma que los neorrealistas ven al DI como un "epifenómeno", ya que su cumplimiento es una mera coincidencia entre éste y los intereses de los Estados. ${ }^{26}$

Para los neorrealistas o realistas funcionales, la sociedad internacional es anárquica y los compromisos internacionales, siempre que no representen un interés para el Estado, se les cumple a través de la fuerza. ${ }^{27}$ Esta afirmación encuentra su principal falencia en que, en las relaciones diarias de la comunidad internacional, el recurso a estos métodos para asegurar el cumplimiento de las obligaciones internacionales no es la regla general, sino que es una excepción. En su extremo, esta teoría puede llegar a ser negacionista al no reconocer el carácter de derecho del DI por no contar con mecanismos de ejecución forzada. Así, no es la fuerza del DI lo que los obliga:Velasco llega a afirmar que las instituciones internacionales no reflejan más que los intereses de los Estados más poderosos, ${ }^{28}$ anulando así la noción de una verdadera cooperación internacional. En este sentido, Engstrom señala que, según el realismo, el DI funcionará si los Estados más poderosos impulsan su cumpli-

24 Barbé, Esther, Relaciones internacionales, Madrid, Tecnos, 1995, pp. 60-65; Guzmán, Andrew T., How International Law Works?, Reino Unido, Oxford University Press, 2010, pp. 16 y ss.; Velasco, Shelom, op. cit., pp. 31-33.

25 Koh, Harold, op. cit., p. 2632; McDougal, Myres S. et al., op. cit., pp. 112 y 113; Raustiala, Kal y Slaughter, Anne-Marie, "International Law, International Relations and Compliance (Princeton Law \& Public Affairs Paper No. 02-2)”, en Carlnaes, Walter et al. (eds.), The Handbook on International Relations, Londres-Thousand Oaks-Nueva Delhi, Sage Publications, 2002, pp. 538-555.

26 Guzmán, Andrew T., “A Compliance-Based Theory of International Law”, California Law Review, Berkeley, vol. 90, núm. 6, 2002, pp. 1837 y 1838.

27 Beltrán-Cristancho, Mauricio, "El derecho internacional y las relaciones internacionales en el siglo XX”, Estudios Socio-Jurídicos, Bogotá, vol. 4, núm. 2, marzo de 2010, pp. 53, 54 y 61; Salomón, Mónica, "La teoría de las relaciones internacionales en los albores del siglo XXI: diálogo, disidencia y aproximaciones”, Revista Electrónica de Estudios Internacionales, España, núm. 4, 2002 , p. 3.

28 Velasco, Shelom, op. cit., pp. 31-33. 
miento. ${ }^{29}$ Como afirma Hathaway, en el caso del derecho internacional de los derechos humanos, el respeto y garantía de los derechos humanos sería una mera coincidencia ${ }^{30}$ y no el resultado de una real voluntad del Estado.

Sin embargo, una vertiente matizada establece que, incluso en el ámbito de los derechos humanos, los Estados cumplen con sus obligaciones a través de la coerción o presión que ejercen los Estados más poderosos sobre aquellos menos poderosos. En un mundo extremadamente polarizado, como el de la Guerra Fría, la presencia de un "hegemón" podría tener un impacto en el cumplimiento de las decisiones de los tribunales. No obstante, en una realidad con una multipolaridad, la presión de estas grandes potencias no tendría tanto peso al encauzar el cumplimiento de las obligaciones. Cabe hacer una crítica de la aplicación de este enfoque al trabajo de la Corte Interamericana de Derechos Humanos, cuya competencia contenciosa deberá ser aceptada por los Estados, mediante la declaración mencionada en el artículo 62 de la Convención Americana sobre Derechos Humanos. El Estado más poderoso en las Américas es, incontestablemente, Estados Unidos, quien no es parte de la Convención. Siguiendo estrictamente el enfoque de la hegemonía y poder de ciertos Estados, no le será posible a éste ejercer presión sobre el resto de los países (menos poderosos) al no formar parte del subsistema de la Convención. Es así como esta teoría encuentra una falencia en el caso concreto de los sistemas de protección de derechos.

Guzmán propone una explicación para el cumplimiento del DI que recoge elementos de varias teorías antes descritas. El autor asume tres elementos con relación a los Estados: son entes racionales; actúan en función de sus intereses (sin que importe el proceso interno de definición de esos intereses), y están conscientes del impacto del DI en su comportamiento. Para que el Estado cumpla con sus obligaciones internacionales, es preciso que exista un sistema que implique una sanción por incumplimiento. Este sistema que el autor propone debe incluir un mecanismo de sanciones materiales, así como "costos reputacionales" que afectarían la capacidad del incumplidor de llegar

29 Engstrom, Par, "Human Rights: Effectiveness of International and Regional Mechanisms", Oxford Research Encyclopedia of International Studies, Reino Unido, International Studies Association-Oxford University Press, diciembre de 2017, p. 5, disponible en: https: / /oxfordre.com / internationalstudies / view/10.1093/acrefore/9780190846626.001.0001 / acrefore-9780190846 $626-e-214$ ? rskey $=4 n f R U Q \&$ result $=1$.

30 Hathaway, Oona A., "Do Human Rights Treaties Make a Difference?", The Yale Law Journal, Estados Unidos, vol. 111, núm. 8, 2002, pp. 1945 y 1946. 
a acuerdos futuros. ${ }^{31}$ Las sanciones materiales serán fijadas por los propios Estados o por tribunales internacionales con dicha facultad. En el primer escenario, siempre quedará la duda sobre qué sanciones impondrá la parte afectada y, en el segundo, los tribunales serán quienes determinen la sanción adecuada correspondiente. En este sentido, Poertner señala que en el derecho internacional de los derechos humanos no existen realmente incentivos y sanciones que pudieran presionar a los Estados a cumplir con sus obligaciones (sin importar la fuente de la cual provengan). ${ }^{32}$ Asimismo, concluye Hathaway en su artículo sobre la importancia de los tratados de derechos humanos: el derecho internacional de los derechos humanos es un área en la que los Estados tienen pocos incentivos para monitorear el incumplimiento de las normas. ${ }^{33}$

Con respecto a los “costos reputacionales”, la reputación del Estado tendría un valor que se vería afectado al incumplir con sus obligaciones. Para Guzmán, los Estados con “mejores reputaciones” tienen la posibilidad de acceder a mejores y futuros acuerdos en el marco de la comunidad internacional, ${ }^{34}$ lo que impulsaría al cumplimiento del DI al configurarse como un interés del Estado. Para la valoración sobre los costos y beneficios del incumplimiento, se deberá tener en cuenta, según el autor, cuatro elementos: la gravedad de la violación; las razones para la violación; la claridad de la obligación y de la violación, y el conocimiento que el Estado tenía sobre su incumplimiento. ${ }^{35}$ Sobre este punto concuerda Dothan en su estudio sobre los mecanismos que incrementan la legitimidad de los tribunales internacionales. ${ }^{36}$

El incumplimiento implicaría, entonces, una inconsistencia entre los intereses del Estado y el contenido de las normas internacionales, incluidas las sentencias emitidas por tribunales internacionales. ${ }^{37}$ Esta teoría parecería

31 Guzmán, Andrew T., “A Compliance-Based Theory...”, op. cit., pp. 1840-1846.

32 Poertner, Mathias, op. cit., p. 10.

33 Hathaway, Oona A., op. cit., p. 1938.

34 Guzmán, Andrew T., “A Compliance-Based Theory...”, op. cit., p. 1855; Guzmán, Andrew T., How International Law..., cit., pp. 179-219.

35 Guzmán, Andrew T., “A Compliance-Based Theory...”, op. cit., pp. 1861 y 1862.

36 Dothan, Shai, "How International Courts Enhance their Legitimacy", Theoretical Inquiries in Law, Tel Aviv, vol. 14, núm. 2, 2013, p. 459.

37 Elvy, Stacey-Ann, "Theories of State Compliance with International Law: Assessing the African Union's Ability to Ensure State Compliance with the African Charter and Constitutive Act”, Georgia Journal of International and Comparative Law, Estados Unidos, vol. 41, núm. 1, 2012, pp. 75-155; Velasco, Shelom, op. cit., pp. 31-33. 
no tener una respuesta en el caso de los tribunales de derechos humanos, ya que no están claros los beneficios que obtendría el Estado al cumplir con las medidas de reparación ordenadas. Sin embargo, siguiendo el planteamiento de Guzmán, los costos reputacionales del incumplimiento, el oprobio de sus contrapartes, deben ser tenidos en cuenta por parte del Estado al momento de decidir cumplir o no cumplir con sus obligaciones. En este sentido, continuando con el argumento de Guzmán, los beneficios de cumplir con estas sentencias serán reflejados al ser un Estado con un nivel de cumplimiento alto frente a la comunidad internacional, obteniendo ciertos beneficios. ${ }^{38}$

Por otro lado, el institucionalismo es otra de las corrientes que aborda el cumplimiento del DI y, en tal tarea, comparte con el realismo algunos de sus postulados. Por un lado, el institucionalismo mantiene que el Estado, concebido como ente racional, sigue siendo uno de los actores fundamentales y busca su supervivencia en un mundo caótico. Pero, a diferencia de los realistas, los institucionalistas sí consideran que es posible alcanzar la cooperación internacional, ya que puede llegar a ser un interés de los Estados y, por tanto, buscarán encaminar sus actuaciones a alcanzarla. Ellos afirman, asimismo, que las instituciones (normas, prácticas y procesos de toma de decisiones que modifican expectativas) ayudan a alcanzar esa cooperación y pueden influenciar el comportamiento del Estado (principal diferencia con el realismo). ${ }^{39}$

Para esta corriente, el incumplimiento surge de la ineficacia de las instituciones internacionales en su intento de influenciar a los Estados para que cumplan con sus obligaciones internacionales, incluso alejándose de un comportamiento visto como racional. ${ }^{40}$ En esta teoría se plantea la existencia de costos reputacionales que afectan el cumplimiento a largo plazo y de sanciones directas que afectan el cumplimiento a corto plazo. ${ }^{41} \mathrm{Su}$ aplicación al caso del contencioso internacional en materia de derechos humanos es poco factible, pues una de las falencias de esta teoría es que no tiene en cuenta a los actores internos (factor doméstico) ni al tribunal que emite la sentencia como factores que pueden explicar el cumplimiento o incumplimiento de las obligaciones del Estado. En materia de derechos humanos, el rol de las víctimas es esencial para el cumplimiento de las medidas de reparación or-

38 Guzmán, Andrew T., How International Law..., cit., p. 212.

39 Velasco, Shelom, op. cit., pp. 33-35; Voeten, Erik, op. cit., pp. 426 y 427.

40 Engstrom, Par, op. cit., p. 6; Guzmán, Andrew T., How International Law. ., cit., pp. $205-$

207; McDougal, Myres S. et al., op. cit., p. 113; Velasco, Shelom, op. cit., p. 40.

${ }_{41}$ Velasco, Shelom, op. cit., p. 34. 
denadas. Éstas son las destinatarias de la reparación y, como tal, deben tener un rol activo en la implementación de las medidas.

Por otro lado, para el liberalismo (y el neoliberalismo), el Estado, al igual que en las teorías anteriores, es un ente racional que sigue siendo el actor principal en el plano internacional. Sin embargo, existe la posibilidad de un orden mundial pacífico basado en la cooperación internacional y en consideraciones de justicia. El imperio del derecho es la única solución en un mundo caótico y anárquico; pero las normas que lo componen deben ser objetivas y concretas. ${ }^{42}$ Esta teoría pone un especial énfasis en el rol de las organizaciones internacionales en la cooperación y solución pacífica de los conflictos que surgen del incumplimiento de los Estados. A diferencia de las posiciones anteriores, el comportamiento del Estado está determinado por actores políticos y procesos internos, quienes dibujan sus intereses ${ }^{43}$ y que ponen en crisis la supuesta soberanía y el poderío absoluto del Estado en las RI. Así, los intereses de actores no estatales se proyectan en las RI y en el rol del Estado en ese entramado. ${ }^{44}$

En lo referente a las razones por las cuales los Estados cumplen con el DI, el liberalismo (y el neoliberalismo, en parte) pone énfasis en las dinámicas internas de grupos no estatales: el cumplimiento del DI se debe a una estructura interna y a sus defensores. El incumplimiento será el resultado de un fracaso de los actores nacionales en su intento de impulsar el cumplimiento de las obligaciones internacionales como un interés estatal. ${ }^{45}$ En este contexto, propugnan que la democracia es un pilar fundamental en la cooperación internacional para la paz, el bienestar, la justicia y el cumplimiento del DI. ${ }^{46}$ Para Koh, las democracias liberales consolidadas serán menos proclives al conflicto y trabajarán de manera colaborativa a fin de crear un orden mundial pacífico. ${ }^{47}$ Una de las falencias de esta teoría es que el DI no cuenta con mecanismos para medir o controlar esa influencia de los actores internos no estatales. Una de las fortalezas de esta teoría es que sirve para entender los procesos de creación, diseño y funcionamiento de los tribunales internacionales y de la implementación de las medidas de reparación, con participación (o, por lo menos, consideración) de las víctimas.

42 McDougal, Myres S. et al., op. cit., p. 148.

43 Ibidem, pp. 113 y 114; Velasco, Shelom, op. cit., p. 40.

44 Koh, Harold, op. cit., p. 2633.

45 Salomón, Mónica, op. cit., p. 5; Velasco, Shelom, op. cit., p. 40.

46 Hathaway, Oona A., op. cit., p. 1953; McDougal, Myres S. et al., op. cit., p. 114.

47 Koh, Harold, op. cit., p. 2633. 
Por otro lado, entre lo que Hathaway llama "corrientes normativas", ${ }^{48}$ se encuentra el modelo de la legitimidad (fairness model) (que, podría afirmarse, toma elementos del neorracionalismo y del neoliberalismo). Se presume que los Estados sólo cumplirán aquella obligación que esté contenida en una norma que sea el resultado de un proceso previamente establecido y válido. Para Franck, existen cuatro factores que determinarán el cumplimiento de la obligación internacional: la claridad de la norma que contiene la obligación; la validación simbólica (a través de la presencia de rituales o prácticas que le darán validez); la coherencia (relación entre principios racionales y la norma que contiene la obligación), y la adherencia (en relación con la conexión entre la norma y las normas secundarias que se usan para aplicarla e interpretarla).$^{49}$ Con respecto a esto, la crítica que Guzmán presenta tiene que ver con el hecho de que Franck no dilucida realmente cuál es el efecto que tiene la legitimidad sobre por qué los Estados cumplen sus obligaciones. Para Guzmán, lo que falta es un nexo causal entre la legitimidad de la norma y su cumplimiento. ${ }^{50}$ Hathaway menciona que, en el caso del derecho internacional de los derechos humanos, el elemento fuerte es la validación simbólica ${ }^{51}$ de una supuesta universalidad, si no de los derechos en sí, de la existencia de unos valores supremos que la comunidad internacional considera como obligatorios para todos sus miembros.

El constructivismo, a diferencia de las corrientes anteriores, se enmarca en una concepción no racionalista del DI. A pesar de ser todavía un actor fundamental, el Estado no tiene intereses preexistentes que direccionan su comportamiento, sino que éstos se crean a través de su interacción con otros actores nacionales e, incluso, internacionales. El enfoque de estos teóricos es el contexto social de cada uno de los Estados. ${ }^{52}$ Así, el rol de los actores no estatales es fundamental, ya que son éstos quienes influencian directamente la formación del DI, empujando el cumplimiento de sus propios objetivos. En este sentido, tanto el cumplimiento como el incumplimiento de las obli-

48 Estas corrientes están basadas en la concepción de que ni el realismo ni el liberalismo ni el institucionalismo toman en cuenta el valor persuasivo que tienen las normas. Véase Hathaway, Oona A., op. cit., pp. 1955-1960.

49 Franck, Thomas, "Legitimacy in the International System", The American Journal of International Law, Estados Unidos, vol. 82, núm. 4, 1988, pp. 705-759; Raustiala, Kal y Slaughter, Anne-Marie, op. cit., p. 540.

50 Guzmán, Andrew T., “A Compliance-Based Theory...”, op. cit., pp. 1834 y 1835.

51 Hathaway, Oona A., op. cit., p. 1959.

52 Salomón, Mónica, op. cit., p. 40. 
gaciones, así como las RI en sí, son socialmente construidos. ${ }^{53}$ Por ende, el incumplimiento se deberá, en palabras de Velasco, a una débil construcción social que no logró posicionar al cumplimiento de las obligaciones internacionales como un eje fundamental de las RI del Estado. ${ }^{54}$

Todas las corrientes mencionadas aceptan la existencia de actores que influencian el cumplimiento de las obligaciones del Estado, pero cada una de ellas les otorga mayor o menor peso; sin embargo, casi no ponen énfasis en el rol de los propios tribunales internacionales. Para el realismo, las cortes internacionales no cuentan con un poder independiente $y$, por ende, no pueden obligar al Estado a cumplir con sus decisiones. El institucionalismo nunca pone en entredicho la soberanía del Estado y la voluntad de éste al momento de decidir acatar o no las decisiones de las cortes internacionales. Por su parte, el liberalismo y el constructivismo, si bien admiten que las instituciones internacionales pueden influenciar el comportamiento del Estado, proponen que las cortes internacionales no pueden influenciar las interacciones entre el Estado y los actores no estatales; ponen énfasis en las relaciones entre estos dos últimos, y no toman en cuenta las relaciones que pueden existir con las cortes. $^{55}$

Frente a estas teorías clásicas, existe una serie de acercamientos críticos desde las teorías feministas, poscoloniales, ecologistas, entre otras, que ponen en duda las concepciones realistas e institucionalistas sobre el poder y el rol del Estado frente al DI. ${ }^{56}$ En la sección siguiente se pone énfasis en dos corrientes teóricas y su acercamiento al porqué del cumplimiento del DI.

\section{NUEVOS ENFOQUES TEÓRICOS DEL DERECHO \\ INTERNACIONAL PÚBLICO}

\section{Los Critical Legal Studies}

Los Estudios Legales Críticos o Critical Legal Studies (CLS, por sus siglas en inglés) nacieron en Estados Unidos, en la década de 1970, como una crítica

53 McDougal, Myres S. et al., op. cit., p. 113.

54 Velasco, Shelom, op. cit., pp. 37 y 38.

55 Ibidem, pp. 39-43.

56 Bianchi, Andrea, op. cit., pp. 183-310; Salomón, Mónica, op. cit., pp. 23-37. 
Esta revista forma parte del acervo de la Biblioteca Jurídica Virtual del Instituto de Investigaciones Jurídicas de la UNAM

a las teorías jurídicas dominantes y como un proceso de reivindicación de luchas sociales propias de esa década y la anterior. ${ }^{57}$ Para Molina Ochoa, "los crits son el producto de una generación que creció con las esperanzas emancipadoras y revolucionarias de los sesenta, pero que se volvió escéptica frente al papel transformador que podían tener ciertas instituciones jurídicas como los derechos humanos o el Estado de derecho". ${ }^{58}$

Los CLS abarcan diversas propuestas teóricas sin llegar a constituirse como una propuesta teórica independiente y englobante. A pesar de que Russell ensaya una división dentro de los CLS en tres escuelas (la Escuela de Frankfurt de Marxismo crítico; la del Marxismo científico u ortodoxo, y la Escuela de la Perspectiva de las Ciencias Sociales), llega a afirmar que lo que les une es una oposición común a las corrientes tradicionales vigentes. ${ }^{59}$ Concuerda Núñez Vaquero al afirmar que lo que tienen en común los doctrinarios/as de los CLS es un "antagonismo compartido", ${ }^{60}$ principalmente ante el positivismo (liberalismo jurídico) y el realismo (a pesar de que de éste obtienen ciertas categorías de análisis).

Más allá de las diferencias entre los distintos abordajes teóricos, existen puntos coincidentes, ${ }^{61}$ como se presenta a continuación. Los CLS están basados en una crítica a la supuesta neutralidad y objetividad del derecho. En palabras de McDougal, Lasswell y Reisman, el "derecho es político"62 y, por lo tanto, los CLS se oponen a la idea de una aplicación mecánica y objetiva del derecho. ${ }^{63}$ Las sentencias ya no son "textos inmaculados", ${ }^{64}$ sino que son

57 Molina Ochoa, Andrés, "Estudios críticos del derecho”, en Fabra Zamora, Jorge y Núñez Vaquero, Álvaro (eds.), Enciclopedia de filosofía y teoría del derecho, México, UNAM, 2015, vol. 1, pp. 440 y 441, disponible en: https: / / archivos.juridicas.unam. mx/www/bjv/libros/8/3875/ 15.pdf.

58 Ibidem, p. 442.

59 Miljiker, María Eva, "Duncan Kennedy y la crítica a los derechos", Revista Jurídica de la Universidad de Palermo, Buenos Aires, vol. 7, núm. 1, 2006, p. 92; Russell, J. Stuart, "The CLS Challenge to Contemporary Mainstream Legal Philosophy”, Ottawa Law Review, Ottawa, núm. 18, 1986, pp. 1, 4, 5 y 9.

60 Núñez Vaquero, Álvaro, "Teorías críticas del derecho: observaciones sobre el modelo de ciencia jurídica”, Anuario de Filosofía del Derecho, España, núm. 26, 2010, p. 418.

61 Hunt, Alan, “The Theory of Critical Legal Studies”, Oxford Journal of Legal Studies, Reino Unido, vol. 6, núm. 1, 1986, pp. 1-45.

62 Frankenberg, Günter, “Teoría crítica”, Academia. Revista sobre Enseñanza del Derecho, Buenos Aires, año IX, núm. 17, 2011, p. 79; McDougal, Myres S. et al., op. cit., p. 135.

63 Miljiker, María Eva, op. cit., pp. 91 y 92.

64 Molina Ochoa, Andrés, op. cit., p. 443. 
el resultado de un razonamiento jurídico que está permeado por el bagaje cultural y las ideas preconcebidas de los operadores de justicia. La doctrina legal es un mal ensayo de una racionalización de la realidad donde ocurren los hechos. Esto, para los CLS, permite configurar una herramienta política de litigio estratégico. Los CLS retomaron del realismo jurídico la noción de la indeterminación de la norma jurídica. ${ }^{65}$ Los realistas ya propugnaban, como se mencionó anteriormente, que la norma no es objetiva, sino que un mismo postulado puede resultar en interpretaciones diferentes e, incluso, contradictorias. Sin embargo, a diferencia de esta corriente, los CLS fundamentan esta indeterminación no sólo en las propias falencias del lenguaje, sino también en una contradicción fundamental. ${ }^{66}$

En lo que respecta al DI, los CLS no han ahondado en el porqué de su cumplimiento (o incumplimiento), sino que se han enfocado en su naturaleza y objeto. Por un lado, al igual que para el derecho interno, los CLS se oponen al positivismo en el DI y su "campo de batalla" son las fuentes del DI. ${ }^{67}$ Para McDougal, Lasswell y Reisman, los CLS han aportado a nivel conceptual, metodológico y estratégico a esta rama del derecho. En este sentido, han resaltado la importancia de la cultura para entenderlo y cuestionan el carácter absoluto de la soberanía estatal. ${ }^{68}$ Desde lo estratégico, los CLS presentan proyectos radicales de cambio del DI; sin embargo, desde lo metodológico, mantienen códigos binarios para su estudio y consideran al Estado como unidad fundamental de análisis (características de las corrientes tradicionales del pensamiento jurídico).$^{69}$

A pesar de sus evidentes aportes, a los CLS se les critica que pasaron por una etapa de destrucción de categorías tradicionales, pero no aportaron una solución al sistema jurídico moderno. Para Molina Ochoa, los CLS son una "teoría dedicada al diagnóstico" ${ }^{70}$ Concuerda Miljiker al afirmar que los CLS implican una pérdida de fe en el discurso jurídico sin proponer una nueva fe. ${ }^{71}$

65 Miljiker, María Eva, op. cit., p. 91.

66 Núñez Vaquero, Álvaro, op. cit., pp. 418 y 419; Russell, J. Stuart, op. cit., pp. 15 y 16.

67 Carty, Anthony, "Critical International Law: Recent Trends in the Theory of International Law”, European Journal of International Law, Florencia, vol. 2, núm. 1, 1991, p. 1, disponible en: http: / / www.ejil.org/pdfs/2/1 /2026.pdf; Frankenberg, Günter, op. cit., p. 78.

68 McDougal, Myres S. et al., op. cit., p. 146.

69 Carty, Anthony, op. cit.

70 Molina Ochoa, Andrés, op. cit., p. 452.

71 Miljiker, María Eva, op. cit., p. 96. 
En lo que atañe a este ensayo, es importante rescatar de los CLS la valoración del trabajo argumentativo de los/as jueces/zas y la inclusión de consideraciones extralegales como parte de la actuación de los tribunales de derechos humanos. En este sentido, las medidas de reparación ordenadas responderán a un balance entre la norma y la realidad, teniendo en cuenta las particularidades de las víctimas. Sin embargo, como ya se mencionó en párrafos anteriores, estas corrientes no brindan una respuesta a la pregunta de por qué los Estados cumplirían con estas obligaciones. Adicionalmente, a pesar de brindar un espacio a nuevos actores, los CLS todavía presentan un escenario Estado-céntrico del DI.

\section{Los TWAIL}

Los Acercamientos del Tercer Mundo al Derecho Internacional o Nuevos Enfoques del Tercer Mundo al Derecho Internacional (o TWAIL, por sus siglas en inglés ${ }^{72}$ surgieron en la década de 1950 con la llegada de la última ola de independencias después de la Segunda Guerra Mundial. A pesar de su larga trayectoria, aún permanece una discusión sobre si los TWAIL corresponden a una teoría o a una metodología de análisis. Para McDougal, Lasswell y Reisman, Eslava y Pahuja, los TWAIL no son un método ni una teoría, sino un "grupo estratégico comprometido políticamente [y] definido por un grupo común de preocupaciones" (traducción propia). ${ }^{73}$

Por su parte, Okafor manifiesta que, si se entiende que una teoría describe un fenómeno natural o social a través de un método, los TWAIL describen el DI de forma "predecible, lógica y comprobable": buscan predecir, de forma lógica, cómo el DI actuará en relación con el tercer mundo (sur global) y sus necesidades. Los TWAIL proponen, según el autor, diferentes marcos y modelos a través de los cuales buscan entender un fenómeno (DI), convirtiéndose en un conjunto de teorías que conforman una escuela de pensamiento. ${ }^{74}$

72 Si bien existe una crítica a la noción tradicional de "tercer mundo", se puede afirmar que, actualmente, hace referencia al sur global.

73 Eslava, Luis y Pahuja, Sundhya, "Between Resistance and Reform: TWAIL and the Universality of International Law”, Trade, Law and Development, Jodhpur, vol. 3, núm. 1, 2011, p. 104; McDougal, Myres S. et al., op. cit., p. 206.

74 Frankenberg, Günter, op. cit., p. 81; Okafor, Obiora Chinedu, "Critical Third World Approaches to International Law (TWAIL). Theory, Methodology, or Both?”, International Community Law Review, Países Bajos, vol. 10, núm. 4, 2008, pp. 372-377. 
Bianchi, incluso, los incluye en su libro referente a las teorías sobre DI como una de las nuevas teorías relativas a esta rama del derecho. ${ }^{75}$

Mediante un desafío a la narrativa dominante y hegemónica, los TWAIL forman una red heterogénea, policéntrica y dispersa que puede ser calificada como un grupo "sólidamente unido". ${ }^{76}$ En el centro está la oposición a un sistema legal injusto que no toma en cuenta las necesidades del sur global, conformándose así en un "movimiento de coalición para combatir [su] impotencia y victimización". ${ }^{77}$

De manera académica, es posible hacer dos clasificaciones de los TWAIL. La primera de ellas es de carácter histórico y presenta dos o tres TWAIL. Los TWAIL I nacen en la década de 1950 y se desarrollan hasta la década de 1980. Con el fin de la época colonial, el abordaje está basado en la existencia de nuevos Estados independientes que buscan un DI compuesto por reglas más equitativas. Estos TWAIL están conformados no sólo por académicos, sino también por activistas que ponen consideraciones de orden moral y ético en el centro de la discusión. Por su parte, los TWAIL II se consolidan en la década de 1990 y están centrados en las nuevas formas de hegemonía que se han generado y buscan transformar el sistema internacional liberal. Los TWAIL II están compuestos por un gran número de académicos que se enfocan en temas epistemológicos y los procesos de toma de decisiones. Finalmente, para McDougal, Lasswell y Reisman, existe un TWAIL III, que ha tomado fuerza desde el siglo XXI, que es más flexible y enfoca su crítica en la historia de formación del DI y en sus promesas incumplidas, sin perder fe en su poder de cambio. $^{78}$

La segunda clasificación de los TWAIL tiene que ver con la función que se otorga al DI para alcanzar los objetivos planteados anteriormente. Así, existe un sector conservador que no encuentra un enfrentamiento radical entre el derecho y el sistema, ya que, en palabras de Eslava y Pahuja, la

75 Bianchi, Andrea, op. cit.

76 Frankenberg, Günter, op. cit., p. 81; McDougal, Myres S. et al., op. cit., p. 206; Thuo Gathii, James, "TWAIL: A Brief History of its Origins, its Decentralized Network, and a Tentative Bibliography", Trade, Law and Development, Jodhpur, vol. 3, núm. 1, 2011, p. 34.

77 Mutua, Makau, "What Is TWAIL?", Proceedings for the ASIL 94th Annual Meeting, Reino Unido, vol. 94, 2000, p. 37; Ramina, Larissa, "TWAIL - «Third World Approaches to International Law» and Human Rights: Some Considerations”, Revista de Investigações Constitucionais, Curitiba, vol. 5, núm. 1, 2018, p. 263.

78 McDougal, Myres S. et al., op. cit., pp. 210-216 y 223-225. 
justicia no es su preocupación inmediata o se encuentran en una "zona de confort”, en la cual se sienten cómodos. Para este grupo, el DI requiere reformas mínimas, pues las promesas que éste trae podrán solucionar los problemas del sur global. Otro sector es el reformista, cuya propuesta se centra en cambios sustanciales que, sin embargo, no implican la destrucción del sistema internacional como tal. Los TWAIL de este sector son, para los autores, "progresistas estratégicos" o "pragmáticos comprometidos". Finalmente, el sector revolucionario considera que el sistema legal actual no trae consigo justicia y busca una destrucción total del DI tal y como está concebido. Eslava y Pahuja los clasifican como "idealistas comprometidos" o "visionarios confrontativos". ${ }^{79}$

Los TWAIL, entonces, son un conjunto de acercamientos teóricos y prácticos al DI que están fundados en la búsqueda de una "resistencia frente a la proyección de autoridad militar, cultural y económica" que afecta directamente al sur global. ${ }^{80}$ En su seno hay una diversidad de voces que imposibilita hablar de un solo TWAIL; pero, a pesar de la existencia de este "coro de voces", ${ }^{81}$ existen ciertos fundamentos en los cuales se basan los acercamientos teóricos.

El primero de ellos es el reconocer que, a pesar de que el DI se presenta como universal, es eurocéntrico (esta afirmación es compartida por autores clásicos como Shaw y Herdegen) ${ }^{82}$ además, al estar basado en complejos de superioridad del llamado primer mundo, crea un sistema jerárquico basado en la construcción de la otredad, que se traduce en la consolidación de "formas hegemónicas de poder en el DI" ${ }^{83}$ que buscan o que resultan en la opresión del sur global. Otro elemento coincidente de los TWAIL es considerar al colonialismo y su legado como una de las herramientas para favorecer el control de algunos Estados frente a países del sur global. La pobreza y el mal llamado subdesarrollo serían consecuencias directas del proceso de colonización. ${ }^{84}$ Otro elemento de estudio es la normativa, concebida como una herramienta para impulsar intereses dominantes.

79 Eslava, Luis y Pahuja, Sundhya, op. cit., pp. 113 y 114.

80 Thuo Gathii, James, op. cit., p. 36.

81 McDougal, Myres S. et al., op. cit., p. 207.

82 Herdegen, Matthias, op. cit., pp. 14-26; Shaw, Malcom N., International Law, 6a. ed., Reino Unido, Cambridge University Press, 2008, pp. 27-31.

83 McDougal, Myres S. et al., op. cit., p. 207.

84 Eslava, Luis y Pahuja, Sundhya, op. cit., p. 105; Thuo Gathii, James, op. cit., p. 38. 
Al igual que en ciertas corrientes tradicionales, los TWAIL critican la indeterminación de esa norma. Asimismo, ellos señalan que el derecho no es apolítico y que las fronteras entre lo político y la norma deben eliminarse para entender el fenómeno jurídico como un instrumento de cambio. ${ }^{85}$ Frente a esto, se reconoce que los desbalances de poder y las inequidades que afectan al mal llamado tercer mundo influyen en la forma en que se producen y desarrollan los conceptos tradicionales del DI. En función de esto, Eslava y Pahuja señalan que es preciso rediseñar no sólo conceptos, sino también espacios en los que tradicionalmente se creía que el DI no tenía cabida. ${ }^{86}$ Así, según Mutua, los TWAIL tienen tres objetivos: el primero es entender y deconstruir el DI concebido como un "medio para la creación y perpetuación de un sistema jerarquizado de normas e instituciones que subordinan a los no europeos"; el segundo es construir una alternativa para la gobernanza, y el tercero es colaborar para la erradicación del "subdesarrollo" del sur global. ${ }^{87}$

Es posible concluir que los TWAIL consideran al DI como "prácticas concretas que se expresan en el mundo material, así como un proyecto normativo o ideológico" (traducción propia). ${ }^{88}$ El DI tiene presencia en el día a día y es preciso concebirlo como una herramienta para la "construcción o reconstrucción de lugares, sujetos y modelos de administración”. ${ }^{89}$ En este sentido, el DI se convierte en un campo de batalla para lograr cambios sustanciales en las comunidades y pueblos del llamado tercer mundo. Sin embargo, en muchas de las discusiones académicas de TWAIL, las cuestiones sobre la naturaleza del DI están prácticamente ausentes de manera directa, aunque de manera indirecta hay alusiones a estos temas.

Desde los TWAIL, incluso el derecho internacional de los derechos humanos está basado en "una retórica y un corpus arrogante eurocéntrico", ${ }^{90}$ que impide establecer claramente la relación entre universalidad y particularidad. En este sentido, los derechos humanos no son ni objetivos ni apolíticos, sino que, en palabras de Ramina, se convierten en una forma de "im-

\footnotetext{
85 Frankenberg, Günter, op. cit., pp. 81-83.

86 Eslava, Luis y Pahuja, Sundhya, op. cit., pp. 109-111.

87 Mutua, Makau, “What Is...”, op. cit., p. 31.

88 Eslava, Luis y Pahuja, Sundhya, op. cit., p. 122.

89 Ibidem, pp. 123-125.

90 Mutua, Makau, "What Is...”, op. cit., p. 36.
} 
poner estándares europeos"91 a través de la "metáfora del salvaje, la víctima y el salvador". ${ }^{92}$ Incluso, los mecanismos supranacionales de protección de derechos humanos, como los creados en el marco de la ONU, ${ }^{33}$ ayudarían a consolidar estas prácticas de imposición de criterios eurocéntricos. Estos criterios, se podría afirmar, están plasmados en la determinación de la responsabilidad del Estado, así como en las obligaciones impuestas como mecanismos de reparación de las violaciones.

A pesar de que se admite que esta corriente de pensamiento está centrada en la persona, los pueblos y sus necesidades, el Estado, al igual que en las corrientes teóricas tradicionales, sigue siendo un actor fundamental y objeto principal de estudio. Sin embargo, cabe señalar que existe un cambio esencial en el rol que éste tiene en relación con las personas. ${ }^{94}$ Esto se traduce, a nuestro parecer, con la concepción del Estado como titular de obligaciones frente a la persona.

Así, a través de los planteamientos de los TWAIL, es posible abordar el cumplimiento de las obligaciones internacionales del Estado contenidas en las sentencias de los tribunales de derechos humanos como un instrumento de lucha en el día a día que permite deconstruir conceptos y lugares con el fin de responder a las necesidades de las víctimas de violaciones a los derechos humanos. El DI ya no es una burbuja lejana, impuesta, sino que se construye con las víctimas, los usuarios/as de los tribunales y los titulares de obligaciones (Estados). Sin embargo, no es posible determinar por qué los Estados cumplirían con las sentencias provenientes de los tribunales de derechos humanos.

\section{CONCLUSIÓN}

A lo largo de este artículo, se planteó una breve revisión del abordaje del cumplimiento de las obligaciones internacionales del Estado desde las dife-

91 Ramina, Larissa, op. cit., p. 264.

92 Mutua, Makau, "Savages, Victims, and Saviors: The Metaphor of Human Rights", Harvard International Law Journal, Estados Unidos, vol. 42, núm. 1, 2001, pp. 201-245.

93 McDougal, Myres S. et al., op. cit., p. 207; Mutua, Makau, “What Is...”, op. cit., pp. 31-37; Ramina, Larissa, op. cit., p. 263; Thuo Gathii, James, op. cit., p. 35.

94 Mutua, Makau, "What Is...”, op. cit., pp. 34 y 35. 
rentes corrientes teóricas del DI. En lo que respecta a las teorías que se han denominado como tradicionales, las propuestas están basadas en un modelo Estado-céntrico. Al intentar explicar por qué el Estado cumple con sus compromisos internacionales, sea cual sea la fuente de la que provengan, no llegan a una conclusión clara o presentan explicaciones basadas meramente en la voluntad de los Estados. Esto se refleja no sólo en las teorías jurídicas, sino también en las que abordan el tema desde las RI.

Entre las que hemos llamado nuevas teorías del DI, se encuentran los Critical Legal Studies y los Acercamientos del Tercer Mundo al Derecho Internacional (o Nuevos Enfoques del Tercer Mundo al Derecho Internacional). Si bien éstos proponen una ruptura con la visión tradicional de estudio de esta rama del derecho, mantienen algunas de las categorías analíticas de las corrientes más tradicionales. Por ejemplo, el Estado sigue siendo un actor importante en el marco y, en algunas subcorrientes, el modelo racionalista sigue siendo predominante. Estas corrientes no han puesto énfasis en la discusión teórica sobre el porqué del cumplimiento de las obligaciones internacionales del Estado.

Se ha observado que las principales corrientes filosófico-teóricas relativas al DI no han brindado una respuesta tajante sobre las razones que explicarían la adecuación del comportamiento estatal a sus obligaciones internacionales, especialmente en lo que respecta a aquellas ordenadas por los tribunales internacionales de derechos humanos.

\section{BIBLIOGRAFÍA}

Alter, Karen, “Agents or Trustees? International Courts in their Political Context”, European Journal of International Relations, Nueva York, vol. 14, núm. 1, 2008.

APARICIO, Marco y PisArello, Gerardo, "Los derechos humanos y sus garantías”, en BONET PÉREZ, Jordi y SÁNCHEZ, Víctor (coords.), Los derechos humanos en el siglo XXI. Continuidad y cambios, España, Huygens, 2008.

Austin, John, The Providence of Jurisprudence Determined, Reino Unido, Cambridge University Press, 2010.

Baluarte, David C. "'Strategizing for Compliance: The Evolution of a Compliance Phase of Inter-American Court Litigation and the Strategic Impe- 
Esta revista forma parte del acervo de la Biblioteca Jurídica Virtual del Instituto de Investigaciones Jurídicas de la UNAM

rative for Victims' Representatives", American University International Law Review, Washington D. C., vol. 27, núm. 2, 2012.

BARBÉ, Esther, Relaciones internacionales, Madrid, Tecnos, 1995.

BELTRÁN-CRISTANCHO, Mauricio, "El derecho internacional y las relaciones internacionales en el siglo XX”, Estudios Socio-Jurídicos, Bogotá, vol. 4, núm. 2, marzo de 2010.

BIANCHI, Andrea, International Law Theories. An Inquiry into Different Ways of Thinking, Reino Unido, Oxford University Press, 2016.

CARTY, Anthony, "Critical International Law: Recent Trends in the Theory of International Law”, European Journal of International Law, Florencia, vol. 2, núm. 1, 1991, disponible en: http: / / www.ejil.org/pdfs/2/1/2026.pdf.

DOTHAN, Shai, "How International Courts Enhance their Legitimacy", Theoretical Inquiries in Law, Tel Aviv, vol. 14, núm. 2, 2013.

ELVY, Stacey-Ann, “Theories of State Compliance with International Law: Assessing the African Union's Ability to Ensure State Compliance with the African Charter and Constitutive Act", Georgia Journal of International and Comparative Law, Estados Unidos, vol. 41, núm. 1, 2012.

ENGSTROM, Par, "Human Rights: Effectiveness of International and Regional Mechanisms", Oxford Research Encyclopedia of International Studies, Reino Unido, International Studies Association-Oxford University Press, diciembre de 2017, disponible en: https: / / oxfordre.com/internationalstudies / view / 10.1093 / acrefore / 9780190846626.001 .0001 / acrefore-978019084 6626-e-214? rskey =4nfRUQ\&result $=1$.

ESLAVA, Luis y PAHUJA, Sundhya, "Between Resistance and Reform: TWAIL and the Universality of International Law", Trade, Law and Development, Jodhpur, vol. 3, núm. 1, 2011.

FRANCK, Thomas, "Legitimacy in the International System”, The American Journal of International Law, Estados Unidos, vol. 82, núm. 4, 1988.

FrAnkenberG, Günter, "Teoría crítica”, Academia. Revista sobre Enseñanza del Derecho, Buenos Aires, año IX, núm. 17, 2011.

Grotius, Hugo, The Rights of War and Peace, trad. de Archibald Campbell, Nueva York-Londres, M. Walter Dunne, 2014.

Guzmán, Andrew T., "A Compliance-Based Theory of International Law", California Law Review, Berkeley, vol. 90, núm. 6, 2002.

GuZmán, Andrew T., How International Law Works?, Reino Unido, Oxford University Press, 2010. 
HART, Herbert, El concepto del derecho, trad. de Genaro R. Carrió, Buenos Aires, Abeledo-Perrot, 1963.

Hathaway, Oona A., "Do Human Rights Treaties Make a Difference?", The Yale Law Journal, Estados Unidos, vol. 111, núm. 8, 2002.

HaWkins, Darren y JaCOBY, Wade, "Partial Compliance: A Comparison of the European and Inter-American Courts of Human Rights", Journal of International Law and International Relations, Toronto, vol. 6, núm. 1, 2010.

Henkin, Louis, How Nations Behave: Law and Foreign Policy, 2a. ed., Nueva York, Columbia University Press, 1979.

Herdegen, Matthias, Derecho internacional público, trad. de Marcela Anzola, México, Fundación Konrad Adenauer-UNAM, Instituto de Investigaciones Jurídicas, 2005.

HuneEus, Alexandra, "Compliance with International Court Judgments and Decisions", en Romano, Cesare et al. (eds.), The Oxford Handbook of International Adjudication, Reino Unido, Oxford University Press, 2015.

HunT, Alan, "The Theory of Critical Legal Studies", Oxford Journal of Legal Studies, Reino Unido, vol. 6, núm. 1, 1986.

KLABBERS, Jan, "Constitutionalism and the Making of International Law Fuller's Procedural Natural Law", No Foundations: Journal of Extreme Legal Positivism, Helsinki, núm. 5, 2008.

$\mathrm{KoH}$, Harold, "Why Do Nations Obey International Law?", The Yale Law Journal, Estados Unidos, vol. 106, núm. 8, 1997.

MCDougat, Myres S. et al., "Theories about International Law: Prologue to a Configurative Jurisprudence", Faculty Scholarship Series, Estados Unidos, núm. 2577, 1968, disponible en: http: / / digitalcommons.law.yale.edu/ fs__papers 12577 .

MiljIKeR, María Eva, "Duncan Kennedy y la crítica a los derechos", Revista Jurídica de la Universidad de Palermo, Buenos Aires, vol. 7, núm. 1, 2006. Molina OCHOA, Andrés, "Estudios críticos del derecho", en FABRA ZAMORA, Jorge y NúÑEZ VAquERO, Álvaro (eds.), Enciclopedia de filosofía y teoría del derecho, México, UNAM, 2015, vol. 1, disponible en: https: / / archi vos.juridicas.unam. mx/www/bjv/libros/8/3875/15.pdf.

MutuA, Makau, "Savages, Victims, and Saviors: The Metaphor of Human Rights", Harvard International Law Journal, Estados Unidos, vol. 42, núm. 1, 2001.

MutuA, Makau, "What Is TWAIL?", Proceedings for the ASIL 94th Annual Meeting, Reino Unido, vol. 94, 2000. 
Esta revista forma parte del acervo de la Biblioteca Jurídica Virtual del Instituto de Investigaciones Jurídicas de la UNAM

NúÑEZ VAQUERO, Álvaro, “Teorías críticas del derecho: observaciones sobre el modelo de ciencia jurídica”, Anuario de Filosofía del Derecho, España, núm. 26, 2010.

OKAFOR, Obiora Chinedu, "Critical Third World Approaches to International Law (TWAIL). Theory, Methodology, or Both?", International Community Law Review, Países Bajos, vol. 10, núm. 4, 2008.

PAlOmbinO, Fulvio Maria, "Compliance with International Judgments: Between Supremacy of International Law and National Fundamental Principles", Zeitschrift für ausländisches öffentliches Recht und Völkerrecht, Heidelberg, núm. 75, 2015.

POERTNER, Mathias, "Institutional Capacity for Compliance: Domestic Compliance with the Inter-American Court of Human Rights”, APSA 2013 Annual Meeting Paper, Chicago, 2013, disponible en: https: / /ssrn.com / abst ract $=2299763$.

Posner, Eric A. y Yoo, John C., "Judicial Independence in International Tribunals", California Law Review, Berkeley, vol. 93, núm. 1, 2005.

RAMINA, Larissa, “TWAIL - «Third World Approaches to International Law» and Human Rights: Some Considerations", Revista de Investigações Constitucionais, Curitiba, vol. 5, núm. 1, 2018.

Raustiala, Kal y Slaughter, Anne-Marie, "International Law, International Relations and Compliance (Princeton Law \& Public Affairs Paper No. 022)", en CARlnaes, Walter et al. (eds.), The Handbook on International Relations, Londres-Thousand Oaks-Nueva Delhi, Sage Publications, 2002.

Romano, Cesare, “The Proliferation of International Judicial Bodies: The Pieces of the Puzzle", New York University Journal of International Law and Politics, Nueva York, vol. 31, núm. 4, 1999.

Romero, Alexia y STATON, Jeffrey K., "Clarity and Compliance in the Inter-American Human Rights System" (manuscrito), 2011, disponible en: http: / / biblioteca.cejamericas.org / bitstream / handle/2015 / 448 / Clarity-andCompliance-in-the-Inter-American-Human-Rights-System.pdf? sequence $=1$ \&is Allowed $=y$.

RusSELL, J. Stuart, "The CLS Challenge to Contemporary Mainstream Legal Philosophy”, Ottawa Law Review, Ottawa, núm. 18, 1986.

SALOMÓN, Mónica, "La teoría de las relaciones internacionales en los albores del siglo XXI: diálogo, disidencia y aproximaciones", Revista Electrónica de Estudios Internacionales, España, núm. 4, 2002. 
Esta revista forma parte del acervo de la Biblioteca Jurídica Virtual del Instituto de Investigaciones Jurídicas de la UNAM

SHAw, Malcom N., International Law, 6a. ed., Reino Unido, Cambridge University Press, 2008.

Sorensen, Max, Manual de derecho internacional público, trad. de Dotación Carnegie para la Paz Internacional, México, Fondo de Cultura Económica, 2011.

Thuo GathII, James, "TWAIL: A Brief History of its Origins, its Decentralized Network, and a Tentative Bibliography”, Trade, Law and Development, Jodhpur, vol. 3, núm. 1, 2011.

TOMUSChAT, Christian, "International Courts and Tribunals", en RüDIGER, Wolfrum (ed.), Max Planck Encyclopedia of Public International Law, Reino Unido, Oxford University Press, 2008, disponible en: https: / /opil.ouplaw. com/view/10.1093/law:epil/9780199231690/law-9780199231690-e35? rskey $=W j Z_{W} R 8$ \& result $=6 \& p r d=$ MPIL.

VELASCO, Shelom, "The Inter-American Court of Human Rights: Emerging Patterns in Judgment Compliance", Thesis and Dissertations, Bloomington, núm. 29, 2016, disponible en: https: / /www.repository.law.indiana.edu/cgi/ viewcontent.cgi?article $=1028$ \& context $=$ etd.

VOeten, Erik, "International Judicial Independence", en DunOfF, Jeffrey y POLlaCK, Mark A. (eds.), Interdisciplinary Perspectives on International Law and International Relations: The State of the Art, Reino Unido, Cambridge University Press, 2012. 